\title{
Lifestyle neuropathology: how our behavior harms our brains and what we can do about it
}

\author{
Werner Paulus
}

Published online: 21 December 2013

(C) Springer-Verlag Berlin Heidelberg 2013

The most common neurological disorders, including Alzheimer's disease, Parkinson's disease, stroke and brain injury are not solely the result of fate or unfavorable genetics. In fact, major brain diseases such as Alzheimer's disease and stroke are highly influenced by lifestyle patterns, such as smoking, obesity, education, and reduced cognitive, physical and social activity. Other harmful activities of life lead to brain impairment and pathology per se; these include alcoholism, use of illicit drugs, exposure to environmental toxins, chronic stress, and engagement in sports that feature repeated head impact. In addition, lifestyle factors may alter the relation of pathology to neurological symptoms. For example, more cognitive activity and better education may decrease the probability of dementia for any given level of neuropathology by increasing cognitive reserve.

Beneficial lifestyle choices considerably reduce the risk of developing neurological disease. It has been estimated that $50 \%$ of all Alzheimer's disease might be prevented through reducing important risk factors, i.e., by not smoking, being physically and mentally active, combating low education, and properly treating or preventing chronic diseases and conditions such as hypertension, high cholesterol, diabetes, obesity in mid-life and depression. Similarly, up to $80 \%$ of strokes are preventable by modifying lifestyle factors, such as increasing physical activity, modifying cholesterol levels, eating better, managing blood pressure and blood glucose, losing weight, and stopping smoking.

Given the immense medical, social and economic burden of harmful patterns of lifestyle, it is remarkable that this is an under-investigated area of research in neuropathology and neuroscience. One reason may be that neuroscientists have been educated in the belief that most neuropathological diseases are primarily due to genetic and cellular malfunctions that should be analyzed experimentally using cellular and molecular biological techniques.

Helping to begin to address this deficiency, this issue of Acta Neuropathologica includes a cluster of review papers, each focusing on a behavioral risk factor for neurological disease, including obesity, brain injury induced by sporting activity, smoking, alcoholism, addiction to illegal drugs, stress, and reduced cognitive and social activity. The papers cover clinical and epidemiological data, human neuropathology, neuroimaging and experimental studies on mechanisms.

It is hoped that this cluster of articles will encourage neuropathologists and other neuroscientists with an interest in neurological disease to engage in more interdisciplinary research, combining cellular and molecular biology with behavioral biology, epidemiology and biomarker analysis. Neuropathologists, as neuroscientists with both clinical and experimental expertise, are in a unique position to study experimentally the effects of modifying lifestyles using relevant models of disease and validating the results in clinicopathological studies using human tissues. Furthermore, the papers may stimulate all neuroscientists to contribute to the education of society regarding lifestyles that prevent disease and preserve a healthy brain.
W. Paulus $(\square)$

Institute of Neuropathology, Münster University Hospital, Pottkamp 2, 48149 Münster, Germany

e-mail: werner.paulus@uni-muenster.de 